\title{
QUEBRA DA DORMÊNCIA DA MACIERA (Malus domestica Borkh) EM SÃO JOAQUIM-SC ${ }^{1}$
}

\author{
VERA LÚCIA IUCHI ${ }^{2}$, TAKESHI IUCHI ${ }^{3}$, EMILIO BRIGHENTI $^{4}$, RENATO DITRICH $^{5}$
}

\begin{abstract}
RESUMO - A macieira é uma fruteira de clima temperado que se caracteriza pelo fenômeno de dormência das gemas, necessitando de determinado número de horas de frio $\left(<7,2^{\circ} \mathrm{C}\right)$ para abertura de gemas. No Brasil, São Joaquim-SC, é o local que apresenta as melhores condições climáticas para o cultivo desta espécie. Entretanto, não é correto afirmar que sempre apresenta horas de frio suficientes para satisfazer as exigências das cultivares. Foram desenvolvidos experimentos no ano 1998 e 1999, em microclimas específicos, que vão de 900 a 1400 metros de altitude, no município de São Joaquim - SC, permitindo afirmar que apenas para a cultivar Gala, nos locais mais altos, acima de $1300 \mathrm{~m}$, em anos acima de 2.300 unidades de frio, é dispensada a superação de dormência artificial.
\end{abstract}

Termos para indexação: macieira, superação da dormência, cianamida hidrogenada, óleo mineral.

\section{BREAKING OF BUD DORMANCY APPLE TREES (Malus domestica Borkh) IN SÃO JOAQUIM, SC.}

SUMMARY - The apple culture is a fruit tree of temperate climate which is characterized by the bud dormancy, requiring a certain amount of hours of chilling $\left(<7.2^{\circ} \mathrm{C}\right)$ for bud opening. In Brazil, São Joaquim Country is the region that presents the best climatic conditions for the cultivation of this specie. However, it is not correct to affirm that it always present sufficient chilling requirement to content the cultivars exigency. Two years of experiments in 1998 and 1999 the trial in specific micro-climates from 900 to $1.400 \mathrm{~m}$ in São Joaquim Country lead to afirm except for Gala cultivar adult plant, in years were the number of chilling units exceed 2.300, there is no need to carry out chemical bud break with hydrogen cyanamide.

Index terms: breaking dormancy, hydrogen cyanamide, mineral oil.

\section{INTRODUÇÃO}

A macieira é uma fruteira de clima temperado que se caracteriza pela queda das folhas no final do ciclo vegetativo e conseqüente entrada em dormência. Para que ocorra brotação e floração, as gemas necessitam ser submetidas a um determinado tempo às baixas temperaturas $\mathrm{e}$, segundo a literatura, considerase a soma de frio abaixo de $7,2^{\circ} \mathrm{C}$. No entanto, temperaturas superiores a $7,2^{\circ} \mathrm{C}$ também podem ser efetivas na abertura de gemas, segundo Samish et al. (1967), Erez e Lavee (1971). Além da temperatura, outros fatores, como a luz, têm efeito negativo no acúmulo de frio, de acordo com Erez et al. (1968). Nas regiões onde há falta de frio, assim como no Sul do Brasil, são observadas anomalias fisiológicas, onde as plantas apresentam baixa porcentagem de brotação de gemas laterais, no período prolongado de floração, e pouca formação de gemas floríferas e baixa produtividade de frutos, segundo Iuchi et al. (1987), Iuchi \& Iuchi (1991), Iuchi \& Iuchi (1987), Petri et al. (1996). No Brasil, São Joaquim é o local que apresenta as melhores condições climáticas para o cultivo da macieira, de acordo com Petri, (1986). Entretanto, não é correto afirmar que apresenta horas de frio suficientes para satisfazer as exigências das cultivares, pois poderá variar com os mesmos. O município apresenta diferentes microclimas, em altitudes que vão de 900 a 1.500 metros. Com a devastação das florestas para a retirada da madeira, houve uma mudança nas condições climáticas, apresentando anos cujo número e unidade de horas de frio fica abaixo das necessidades das cultivares Gala e Fuji. Outros fatores que devem ser levados em conta são o vigor e a idade das plantas, exigindo maior ou menor número de horas de frio, segundo Petri et al. (1986). Pomares novos foram e estão sendo plantados, e o porta-enxerto mais utilizado é o Marubakaido, de porte vigoroso, fazendo com que haja uma necessidade de frio para a superação da dormência das gemas. A formação da planta, nos primeiros anos, é de importância fundamental para o sucesso do empreendimento. Uma boa brotação das gemas que permita selecionar ramos bem formados e melhor distribuídos, favorece a penetração de luz, que é de primordial necessidade. Uma melhor brotação das gemas laterais em plantas adultas vai permitir à planta uma melhor formação de órgãos de frutificação. Métodos físicos (Iuchi \& Iuchi, 1991) e químicos podem ser utilizados para complementar o frio necessário à superação de dormência das gemas, de acordo com Petri et al. (1996), Iuchi et al. (1987), Pasqual \& Petri (1979).

$\mathrm{O}$ óleo mineral $(\mathrm{OM})$, a cianamida hidrogenada $(\mathrm{CH})$ e principalmente a ação conjunta desses dois produtos são, hoje, os mais eficientes e os únicos que se encontram registrados para a superação de dormência da macieira (Nee, 1986).

O óleo mineral a 3\% + CH 0,25\% propiciam uma brotação de gemas laterais equivalentes às doses de $\mathrm{CH}$ nas concentrações de 1 a 1,5\%. A concentração de OM poderá variar de 3 a 4\% e da

\footnotetext{
1 (Trabalho 167/2000). Recebido: 03/07/2000. Aceito para publicação: 00/00/2000

2 Eng $^{\text {a }}$ Agra $^{\mathrm{a}}$, DSc, EPAGRI, CP 81, CEP 88600-000 - São Joaquim, SC.

3 Eng $^{\circ}$ Agr $^{\circ}$, DSc, EMBRAPA-JICA, CP 81, CEP 88600-000 - São Joaquim, SC.

4 Eng $^{\circ}$ Agr $^{\circ}$, MSc, EPAGRI, CP 81, CEP 88600-000 - São Joaquim, SC.

$5 \mathrm{Eng}^{\circ}$ Agr $^{\circ}$, MSc, EPAGRI, CP 502, CEP 88034-901 - Florianópolis, SC.
} 
$\mathrm{CH}$ de 0,15 a 0,5\%, dependendo da cultivar e das condições de dormência das plantas (Petri et al., 1996). Segundo Petri et al. (1996) e Iuchi et al. (1987, 1986), a produção por planta nem sempre aumenta com a aplicação de $\mathrm{OM}+\mathrm{CH}$. O uso do raleio deve ser adequado, o que pode interferir nos dados de produção. Outro problema que pode exercer influência na produção, é a polinização deficiente devido à concentração de flores e o aumento no número de cachos florais. Também é importante ressaltar que o efeito benéfico do aumento da brotação de gemas laterais que, na maioria, são gemas vegetativas, será observado principalmente após o segundo ano (Petri (1986), Iuchi et al. 1986).

Com o objetivo de observar a necessidade de uso de agentes químicos sobre a brotação de gemas nas plantas de macieira das cultivares Gala e Fuji, plantas jovens e adultas, em diferentes altitudes do município de São Joaquim, realizou-se este ensaio.

\section{MATERIAIS E MÉTODOS}

Foram conduzidos no município de São Joaquim, nos anos de 1998 e 1999, no total de dezessete experimentos, sendo as cultivares a Gala e Fuji com plantas jovens, que vão de 1 até 4 anos e plantas adultas, acima de 8 anos, ou com a produção estabilizada. Os experimentos variaram em função da idade de plantas jovens e adultas, da localidade, altitude e das cultivares. $\mathrm{O}$ delineamento experimental adotado foi o inteiramente casualizado, com 1 planta por parcela. $\mathrm{O}$ número de repetições foi variável de acordo com a localidade, ano e cultivar.

Os produtos comerciais utilizados foram: Dormex ${ }^{\circledR}(49 \%$ de cianamida hidrogenada), Assist ${ }^{\circledR}(75,6 \%$ de óleo mineral) e Break Thru ${ }^{\circledR}$ (espalhante adesivo não iônico/penetrante do grupo químico dos silicones).

Os tratamentos, em todos os experimentos, foram aplicados no estádio fenológico de gema dormente, uma única vez, conforme recomendação de Petri et al. (1996).

Em cada parcela, foram demarcados 4 ramos do ano para plantas adultas e 3 ramos para plantas novas. As avaliações do número total de gemas do ramo e das gemas brotadas foram feitas 45 dias após a aplicação dos tratamentos.

A produção $\left(\mathrm{kg} \cdot \mathrm{pl}^{-1}\right)$ foi estimada através da contagem do número de frutos por planta e da estimativa do peso médio dos frutos.

As localidades e suas respectivas altitude estudadas no ano 1998 foram: Bentinho $950 \mathrm{~m}$ (baixo), Postinho $1.400 \mathrm{~m}$ (alto), Estação Experimental de São Joaquim 1.415 m (alto) e Campo de Aviação $1.360 \mathrm{~m}$ (alto).

Tratamentos utilizados no $1^{\circ}$ ano, com plantas novas, foram: $0 ; 0,50 ; 0,70$ e $0,90 \%$ de cianamida hidrogenada $+4 \%$ de óleo mineral, e com plantas adultas; $0 ; 0,30 ; 0,50 ; 0,70 \%$ de cianmida hidrogenada $+3 \%$ de óleo mineral.

Em 1999, as localidades e suas respectivas altitudes estudas foram: Corujas $1.360 \mathrm{~m}$ (alto); Luizinho $1.080 \mathrm{~m}$ (baixo) e Várzea 930 m (baixo).

No ano de 1999, os tratamentos utilizados foram: 0; 0,125; 0,25 e $0,375 \%$ de cianamida hidrogenada $+3 \%$ de óleo mineral e $0,25 \%$ de cianamida hidrogenada $+0,03 \%$ de Break Thru $\mathbb{R}+3 \%$ de óleo mineral, para plantas adultas. Para plantas novas, os tratamentos foram os mesmos, apenas com $2 \%$ de óleo mineral. regressão.

Os dados foram submetidos à análise de variância e de

\section{RESULTADOS E DISCUSSÃO}

No ano de 1998, registraram-se 772 horas de frio, que corresponderam a 2660 unidades de frio, enquanto o ano 99 acumulou 1005 horas de frio, que computaram 2377 unidades de frio ajustado ao Norte Carolina modificado.

Ano de 1998:

As plantas jovens da cultivar Fuji, na localidade de Bentinho $(950 \mathrm{~m})$, responderam aos acréscimos nas doses de cianamida hidrogenada + óleo mineral ao nível de 5\% de probabilidade, revelando efeito quadrático (Tabela 1) que estimou o ponto máximo a $0,5664 \%$ de cianamida hidrogenada + $3 \%$ de óleo mineral, com $74,63 \%$ de gemas laterais brotadas. A função quadrática estimada foi: $\mathrm{Yi}=56,79078455+63,018343 \mathrm{Xi}$ $-55,622038 \mathrm{Xi}^{2}$ (Fig.1). Este resultado está de acordo com o de outros autores que encontraram resposta aos agentes químicos de quebra de dormência quando a quantidade de horas de frio foi insuficiente (Iuchi \& Iuchi (1991), Iuchi et al. (1987), Iuchi \& Iuchi (1986), Petri et al. (1996), Pasqual et al. (1978) Petri et al. (1976). Nesta região mais baixa de São Joaquim, o acúmulo de frio não é suficiente para brotação de gemas laterais.

As plantas jovens da cultivar Fuji, na localidade Campo de Aviação (1300 m), uma região alta, revelou efeito linear, ao nível de 5\% de probabilidade (Tabela 1 ). Para cada aumento de $0,10 \%$ de cianamida hidrogenada + óleo mineral, a porcentagem de brotação aumentou em 1,54\%. A equação estimada foi $\mathrm{Yi}=58,08271997+15,418331 \mathrm{Xi}$ (Fig.2). Este resultado concorda que, em plantas novas, há maior necessidade de frio para que ocorra brotação normal (Petri et al. 1996).

As plantas jovens da cultivar Fuji (nova), para a Estação Experimental de São Joaquim(1415m), apresentaram efeito linear, ao nível de 5\% de probabilidade (Tabela1).Para cada aumento de $0,10 \%$ de cianamida hidrogenada + óleo mineral, a \% de brotação aumentou em 2,45\%. A função estimada foi $Y i=63,51783520+$ 2451543296 Xi (Fig.3).O efeito linear obtido no presente experimento revela que, para este local, doses mais altas de C.H. não atingiram o nível fitótoxico para inibir a brotação de gemas laterais.

As plantas adultas da cultivar Fuji, na localidade de Bentinho (950 m), acusou efeito quadrático, ao nível de 5\% de probabilidade (Tabela 2). A função quadrática estimada foi $\mathrm{Yi}=$ $57,66503+100,955056 \mathrm{Xi}-117,878279 \mathrm{Xi}^{2}$ (Fig.4), com 0,4282\% de cianamida hidrogenada $+2 \%$ de óleo mineral, acusando $79,28 \%$ de gemas brotadas. Mesmo em locais mais baixos, o aumento de doses de $\mathrm{CH}$ atingiu nível fitotóxico, o que concorda com o resultado de Iuchi et al. (1987).

A cultivar Gala, em plantas jovens, na Estação Experimental de São Joaquim (1415 m), revelou efeito quadrático, ao nível de $5 \%$ de probabilidade (Tabela 1$)$. A função estimada foi $\mathrm{Yi}=50,795745+87,321123 \mathrm{Xi}-48,972479 \mathrm{Xi}^{2}$ (Fig.5), com o ponto máximo estimado a $0,8915 \%$ de cianamida hidrogenada + $3 \%$ de óleo mineral e $89,72 \%$ de gemas brotadas. O efeito quadrático apresentado por este experimento revela que as doses mais altas de $\mathrm{CH}$ inibiram a brotação de gemas, e resultados similares foram obtidos por Iuchi et al. (1987) e Petri et al. (1996). 
Para a cultivar Fuji, em plantas adultas, na localidade Postinho (1400m), não houve resposta significativa, ao nível de 5\% de probabilidade (Tabela 2).Isto revela que a necessidade de frio foi satisfeita, pois as plantas mais velhas apresentam menor necessidade de frio para que ocorra brotação (Petri et al. 1996).

A cultivar Gala, adulta, no Bentinho ( $950 \mathrm{~m})$, acusou efeito linear, ao nível de $5 \%$ de probabilidade (Tabela 2). Para cada aumento de $0,10 \%$ de cianamida hidrogenada + óleo mineral, a porcentagem de brotação aumentou em $2,378 \%$. A função estimada foi $\mathrm{Yi}=68,039906+23,786915 \mathrm{Xi}$ (Fig.6).Este local, por ser uma região com menor altitude e conseqüentemente com deficiência de frio, os aumentos de doses de $\mathrm{CH}$ promoveram aumentos na brotação; resultados similares utilizando outros sais de dinitro fenóis foram obtidos por Iuchi \& Iuchi (1991), Iuchi et al. (1990),Iuchi \& Iuchi (1986), Iuchi et al. (1985); no entanto, as doses usadas no presente experimento não atingiram níveis fitotóxicos, o que deve estar associado ao alto vigor de plantas.

Para a cultivar Fuji, plantas novas e adultas, respectivamente, aumentos médios de gemas brotadas para doses acima de 0,5 e $0,3 \%$ de cianamida hidrogenada + óleo mineral a 3 e $2 \%$, respectivamente, para as diferentes altitudes representadas pelos locais, são tão pequenos ou mesmo decrescentes que, provavelmente, não são economicamente viáveis.

Para a cultivar Gala, plantas novas e adultas, nas diferentes altitudes representadas pelos diferentes locais, dosagens acima de $0,5 \%$ de cianamida hidrogenada não são viáveis economicamente.

Como este trabalho foi conduzido em um ano com número razoável de unidades de frio, bem acima da média, pode-se observar que plantas adultas da cv. Fuji, na maior altitude estudada, não responderam aos tratamentos; porém, em menores altitudes, foram receptivas. Plantas jovens, desta mesma cultivar, em altitudes maiores e menores, responderam bem aos tratamentos. Para a cultivar Gala, não foi possível comparar plantas jovens e adultas em locais mais altos e mais baixos, mas, nas idades e locais estudados, houve resposta. Isto concorda com dados de Petri (1996), que níveis de cianamida hidrogenada associada ao óleo mineral variaram de 0,15 a $0,5 \%$.

Ano de 1999:

Nas plantas jovens da cultivar Fuji, na localidade de Corujas (1360 m) houve efeito linear dos tratamentos, ao nível de $5 \%$ de probabilidade (Tabela 3 ). A equação estimada foi $\mathrm{Yi}=$ $63,277884+20,808076$ Xi (Fig.7). Para cada aumento de $0,10 \%$ de cianamida hidrogenada, houve um aumento de $2,08 \%$ de gemas brotadas. Isto está associado ao alto vigor de plantas, pois quanto mais nova a planta, maior será o vigor, e quanto mais vigorosa, maior a necessidade de frio (Petri et al. 1996). Já para a cv. Fuji, plantas adultas, não houve efeito (Tabela 4). Este resultado está de acordo que as plantas de ramos de alto vigor exigem maior quantidade de horas de frio para promover a brotação (Petri et al., 1996). A Gala, plantas novas, neste mesmo local, apresentou um efeito quadrático, ao nível de $5 \%$ de probabilidade (Tabela3) $(\mathrm{Yi}=50,449072+166,183709 \mathrm{Xi}-$ $379,312258 \mathrm{Xi}^{2}$ ) (Fig.8). O efeito quadrático apresentado pelo aumento de doses de C.H. revela que a cultivar Gala necessita de menos frio, pois já atingiu níveis tóxicos, provocando diminuição. Já na Gala, plantas adultas, não houve efeito dos tratamentos neste mesmo nível (Tabela 4). O ponto máximo, estimado, foi $0,2198 \%$ de cianamida hidrogenada $+2 \%$ de óleo mineral com $6,87 \%$ de gemas brotadas.

A cultivar Fuji, plantas adultas, neste mesmo ano, na altitude Luizinho $(1080 \mathrm{~m})$, apresentou efeito linear aos tratamentos $\mathrm{Yi}=62,398833+24,704 \mathrm{XI}$ (Fig.9), ao nível de 5\% de probabilidade (Tabela 4), revelando falta de frio. Este resultado está de acordo com Petri et al. (1996) e Iuchi et al. (1990), enquanto, na cultivar Gala, plantas adultas, não houve efeito ao mesmo nível de probabilidade (Tabela 4), revelando que a necessidade de frio foi satisfeita nesta condição.

A Fuji é uma cultivar de maior vigor e, portanto, era de se esperar que sua exigência em frio para quebra de dormência das gemas laterais fosse maior que a Gala.

A cultivar Fuji, adulta, na localidade de Corujas, a $1.360 \mathrm{~m}$ de altitude, não apresentou efeito aos tratamentos (Tabela 4), revelando que o frio foi satisfeito, enquanto esta mesma cultivar, a $1080 \mathrm{~m}$ de altitude, respondeu aos tratamentos, revelando falta de frio. Em maiores altitudes, era de se esperar que, num ano com 2.377 unidades de frio, o fato ocorresse (Empasc, 1986).

A cultivar Gala, plantas adultas, na localidade da Várzea, a $930 \mathrm{~m}$ de altitude, apresentou efeito linear $\mathrm{Yi}=75,89875+$ 26,945 Xi (Fig.10), a 5\% de probabilidade, enquanto esta mesma cultivar, nas Corujas, a $1360 \mathrm{~m}$, não apresentou efeito significativo neste mesmo nível de probabilidade (Tabela 4).

A diferença para a cultivar Gala, adulta, a $930 \mathrm{~m}$, com resposta linear a $1080 \mathrm{~m}$, que não apresentou resposta aos tratamentos, pode ser devida ao maior vigor das plantas na Várzea a $930 \mathrm{~m}$ (Petri et al., 1996).

Observa-se, claramente, que tanto a cultivar quanto a altitude interferem na reação das plantas aos tratamentos e que a interferência dos anos se deve, principalmente, ao número de unidades de frio correspondentes.

Não houve efeito dos tratamentos sobre a produção em nenhum dos locais estudados (Tabelas 3 e 4). Este resultado vem confirmar observações de Petri et al. (1996), que outros fatores interferem sobre este parâmetro. Ressalta-se também que os locais estudados no segundo ano são diferentes dos do primeiro ano e que os efeitos na produção só são observados a partir do $2^{\circ}$ ano (Petri et al. (1996), Petri (1986), Iuchi, $(1987,1986)$ ).

\section{CONCLUSÕES}

1. Para plantas jovens, de Gala ou Fuji, mesmo em regiões mais altas (acima de 1360 m) de São Joaquim, onde existe bastante frio, há necessidade de produtos químicos para a superação da dormência, para aumentar a brotação.

2. Para plantas adultas, nas regiões mais altas (acima de $1360 \mathrm{~m}$ ), não há necessidade de aplicação de produtos químicos para a superação de dormência; já em regiões mais baixas, há necessidade de utilização de produtos para a superação de dormência.

3. Em plantas adultas de Gala, nos locais baixos (menos de 1080 $\mathrm{m}$ ), em plantas de alto vigor, há necessidade de produtos para a superação de dormência; no entanto, em plantas de baixo vigor, não há necessidade de produtos químicos para a superação de dormência.

4. O espalhante adesivo Break Thru não melhora o efeito do óleo mineral+dormex. 
TABELA 1 - Efeito de cianamida hidrogenada $(\mathrm{CH})$ e do óleo mineral $(\mathrm{OM})$ sobre a de brotação de gemas laterais, em macieira, em São Joaquim, 1.415 m, em 1998 nas localidades de Bentinho (950 m), Campo de Aviação (1300 m) e Estação Experimental de São Joaquim (1400 m) em plantas novas de Fuji e Gala.

\begin{tabular}{|c|c|c|c|c|c|}
\hline \multirow[t]{2}{*}{ Tratamentos } & $950 \mathrm{~m}$ & \multicolumn{2}{|c|}{$1300 \mathrm{~m}$} & \multicolumn{2}{|c|}{$1415 \mathrm{~m}$} \\
\hline & Fuji & Fuji & G a la & $\mathrm{Fuji}$ & G a la \\
\hline Testemunha & 56.93 & 57.00 & 74.9 & 62.09 & 50.46 \\
\hline $0.5 \% \mathrm{C} . \mathrm{H} .+4 \% \mathrm{O} . \mathrm{M}$. & 73.34 & 65.79 & 65.8 & 78.64 & 84.84 \\
\hline $0.9 \% \mathrm{C} . \mathrm{H} .+4 \% \backslash \mathrm{O} . \mathrm{M}$. & 67.87 & 71.07 & 68.8 & 83.47 & 91.18 \\
\hline E fe ito $L$ in e a $r$ & $*$ & $*$ & $\mathrm{~N} \mathrm{~S}$ & $*$ & \\
\hline
\end{tabular}

NS- Não significativo

*Significativo ao nível de 5\% de probabilidade

**Significa ao nível de $1 \%$ de probabilidade

TABELA 2 - Efeito de doses de cianamida hidrogenada $(\mathrm{CH})$ e de óleo mineral $(\mathrm{OM})$ sobre a brotação de gemas laterais de macieira no ano 1998 nas localidades de Bentinho a 950 m e no postinho (1400 m), município de São Joaquim, em plantas adultas de cultivares Fuji e Gala.

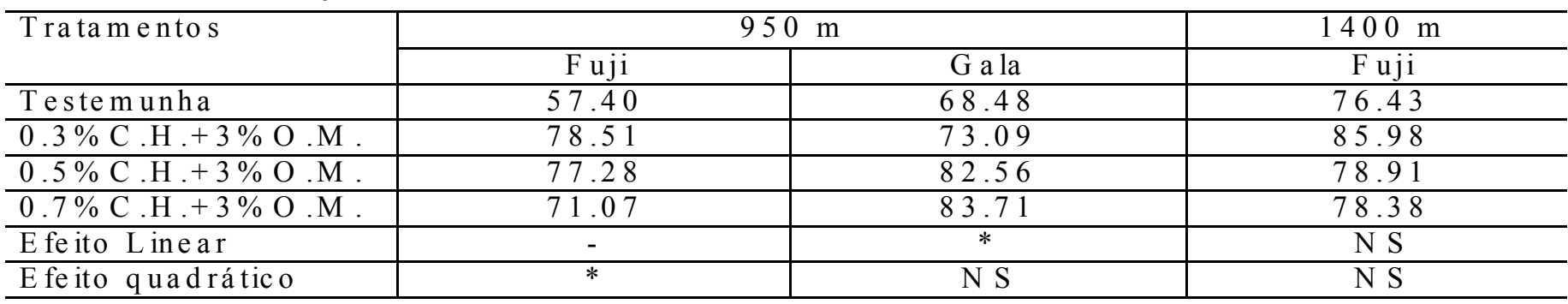

NS- Não significativo

*Significativo ao nível de $5 \%$ de probabilidade

TABELA 3 - Efeito de doses de cianamida hidrogenada $(\mathrm{CH})$ e de óleo mineral $(\mathrm{OM})$ Sobre a brotação de gemas laterais e produção em plantas novas de macieira Fuji e Gala na localidade de Várzea (930 m), Luizinho (1080 m) e na Corujas (1360 m), no município de São Joaquim, no ciclo de 1999/2000.

\begin{tabular}{|c|c|c|c|c|c|c|c|c|}
\hline Tratamentos & \multicolumn{4}{|c|}{ Porcentagem de brotação de gemas laterais } & \multicolumn{4}{|c|}{ Produção de frutos kg/planta } \\
\hline Testemunha & 76.47 & 56.1 & 63.39 & 49.71 & 12.85 & 0 & 48.12 & 29.83 \\
\hline $0.125 \%$ C.H.+3\%O.M. & 76.97 & 58.97 & 66.33 & $67 . .51$ & 15.76 & 0 & 40.76 & 44.17 \\
\hline $0.25 \%$ C.H.+3\%O.M. & 79.68 & 56.38 & 67.85 & 67.18 & 19.05 & 0 & 49.71 & 42.97 \\
\hline $0.375 \%$ C.H.+3\%O.M. & 80.62 & 53.72 & 71.77 & 60.17 & 20.39 & 0 & 45.96 & 48.33 \\
\hline $\begin{array}{l}0.25 \% \\
\text { Thru } 0.03 \%\end{array}$ & 79.49 & 56.4 & 67.93 & 64.44 & 20.2 & 0 & 53.51 & 47.12 \\
\hline
\end{tabular}

NS- Não Significativo

* Significativo ao nível de $5 \%$ de probabilidade

TABELA 4 - Efeito de doses de cianamida hidrogenada $(\mathrm{CH})$ e de óleo mineral (OM) sobre a brotação de gemas laterais e produção em plantas adultas de macieira Fuji e Gala na localidade de Várzea (930 m), Luizinho (1080 m) e na Corujas (1360 m) no município de São Joaquim, no ciclo 1999/2000.

\begin{tabular}{|c|c|c|c|c|c|c|c|c|c|c|}
\hline \multirow[t]{2}{*}{ Tratamentos } & \multicolumn{5}{|c|}{ Porcentagem de brotação de gemas laterais } & \multicolumn{5}{|c|}{ Produção de frutos $\mathrm{kg} /$ planta } \\
\hline & $\begin{array}{c}930 \mathrm{~m} \\
\text { Gala }\end{array}$ & \multicolumn{2}{|c|}{$1080 \mathrm{~m}$} & \multicolumn{2}{|c|}{$1360 \mathrm{~m}$} & $\begin{array}{c}930 \mathrm{~m} \\
\text { Gala }\end{array}$ & \multicolumn{2}{|c|}{$1080 \mathrm{~m}$} & \multicolumn{2}{|c|}{$1360 \mathrm{~m}$} \\
\hline Testemunha & 75.13 & 60.27 & 71.19 & 78.21 & 91.06 & 54.29 & 106.18 & 71.19 & 104.44 & 115.02 \\
\hline $0.125 \%$ C.H.+2\%O.M. & 78.97 & 66.09 & 74.65 & 74.30 & 93.76 & 56.6 & 44.17 & 74.65 & 133.04 & 109.14 \\
\hline $0.1875 \%$ C.H. $+2 \%$ O.M. & - & - & - & 69.28 & 90.48 & - & - & - & 109.89 & 79.78 \\
\hline $0.25 \%$ C.H. $+2 \%$ O.M. & 84.09 & 73.76 & 78.52 & 77.37 & 90.82 & 55.73 & 42.97 & 78.52 & 122.20 & 131.30 \\
\hline $0.375 \%$ C.H.+3\%O.M. & - & - & - & 81.41 & 89.36 & - & - & - & 107.86 & 146.35 \\
\hline Efeito linear & $*$ & * & NS & $*$ & NS & NS & NS & NS & NS & NS \\
\hline Efeito quadrático & NS & & $\mathrm{NS}$ & NS & $\mathrm{NS}$ & NS & NS & NS & NS & NS \\
\hline
\end{tabular}

NS - Não significativo

*Significativo ano nível de $5 \%$ de probabilidade 


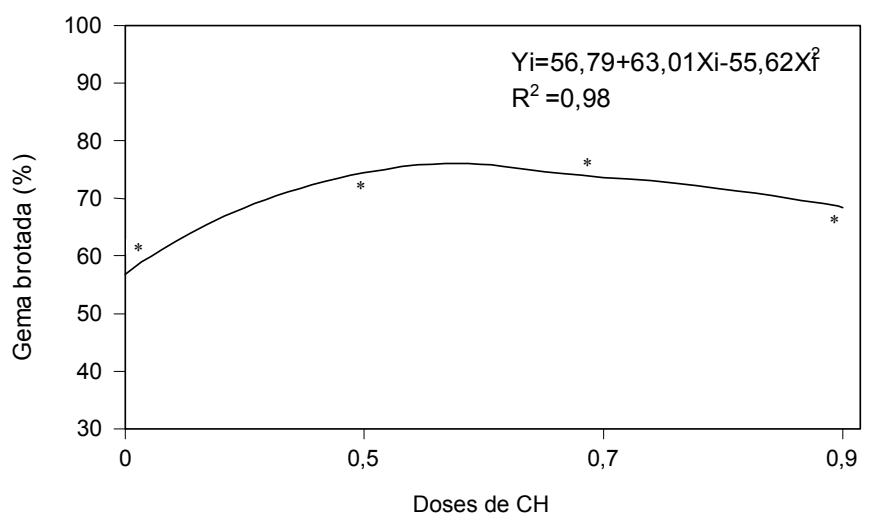

FIGURA 1 - Percentual de gemas axilares brotadas em macieira, cv. Fuji, plantas novas na localidade de Bentinho $950 \mathrm{~m}, 1998$.

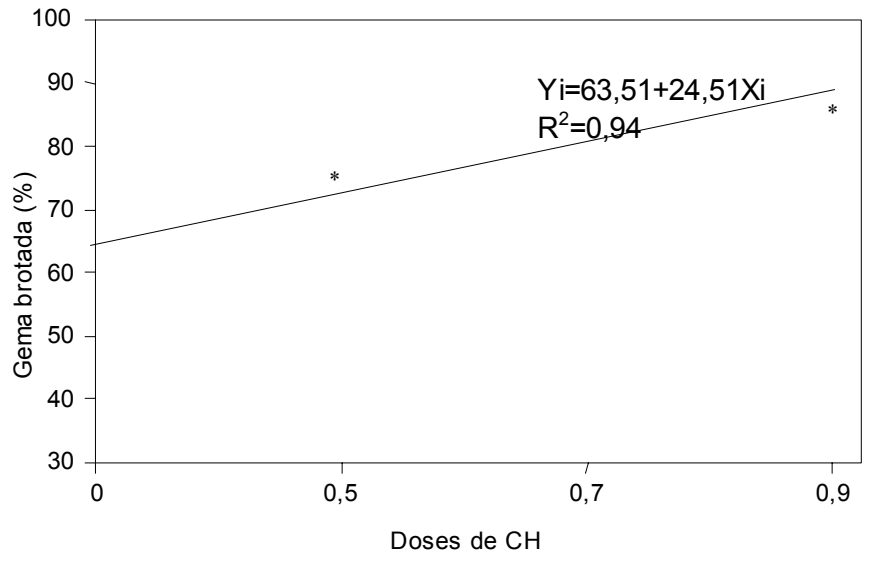

FIGURA 3 - Percentual de gemas laterais brotadas em macieira, cv. Fuji, plantas novas na EESJ, 1415 m, 1998.

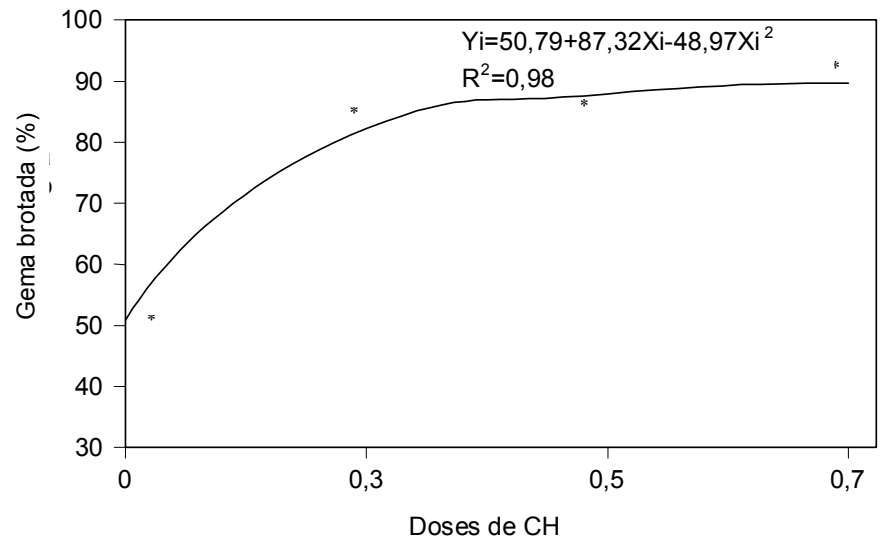

FIGURA 5 - Percentual de gemas laterais brotadas em macieira, cv. Gala, plantas novas, na EESJ 1415 m, 1998.

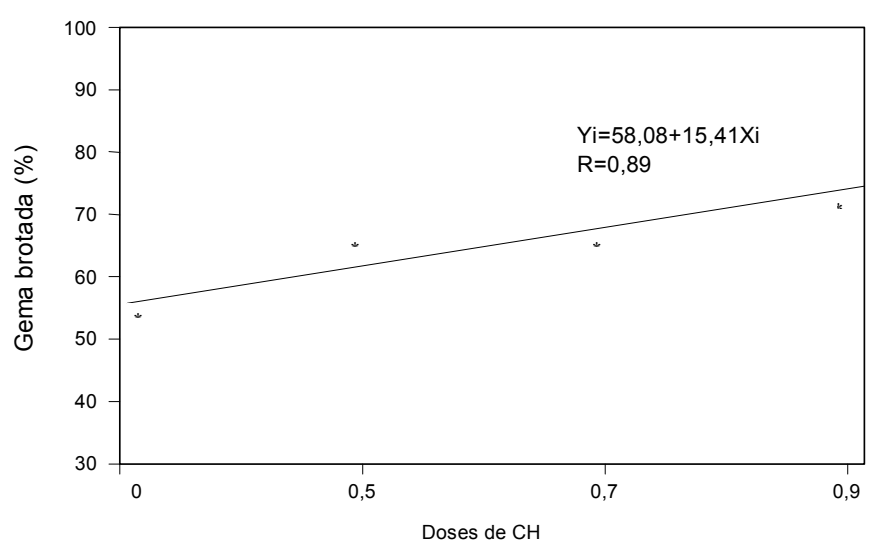

FIGURA 2 - Percentual de gemas laterais brotadas em macieira, cv. Fuji, plantas novas na localidade de Campo de Aviação.

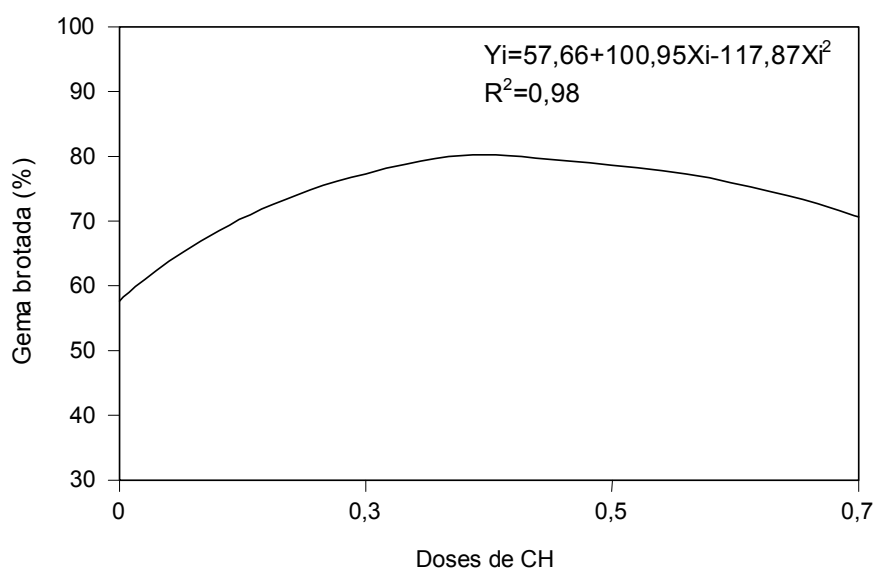

FIGURA 4 - Percentual de gemas brotadas em macieira, cv. Fuji, plantas adulta a $950 \mathrm{~m}$ de altitude, 1998.

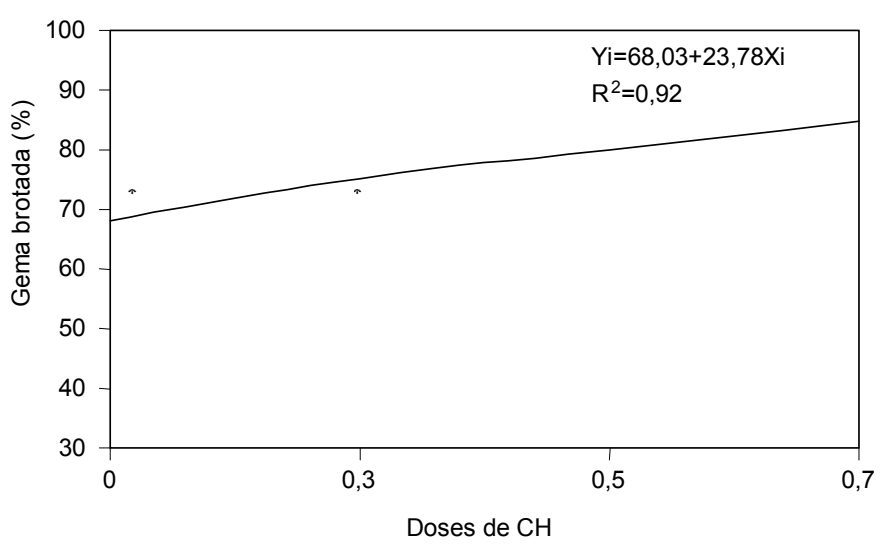

FIGURA 6 - Percentual de gemas laterais brotadas em macieira, cv. Gala, plantas adultas, na localidade de Bentinho 950 m, 1998. 


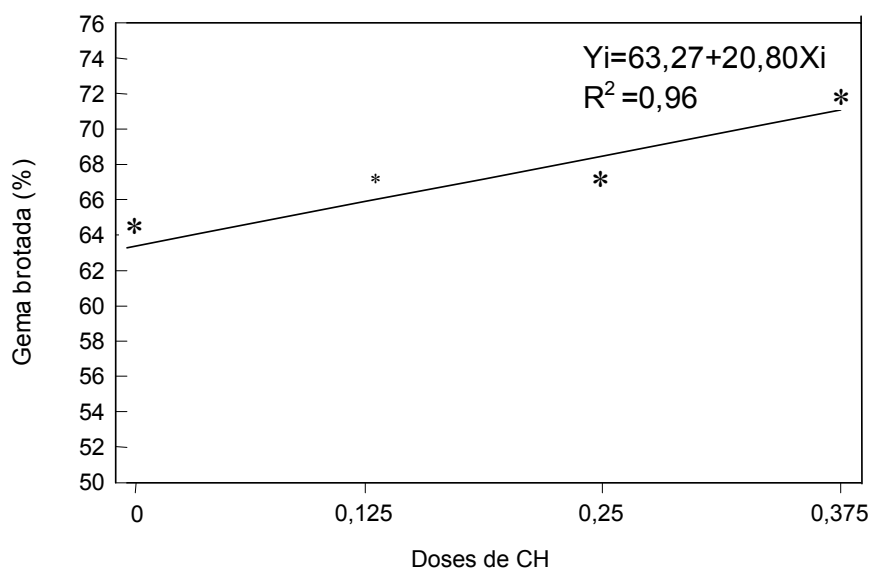

FIGURA 7 - Percentual de gemas laterais brotadas em macieira, cv. Fuji, plantas novas, na localidade de Corujas $1360 \mathrm{~m}$.

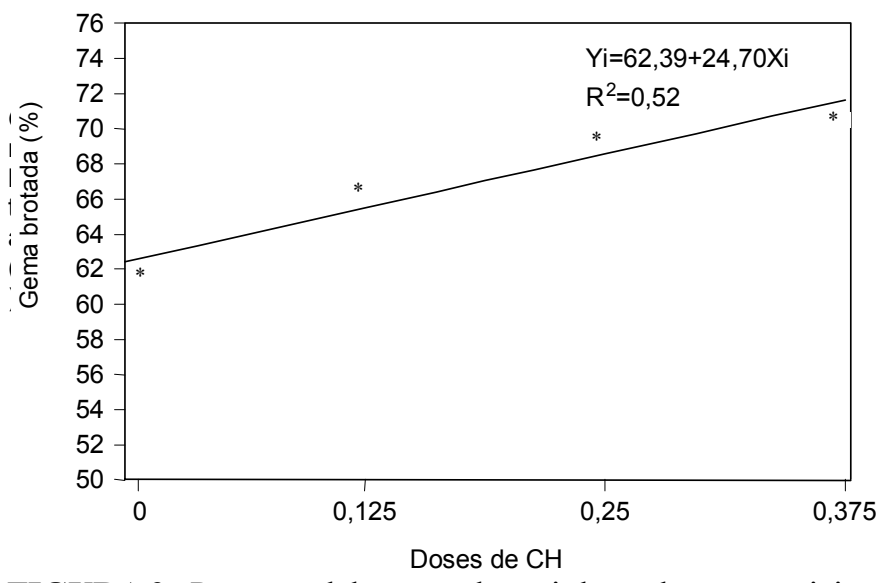

FIGURA 9 - Percentual de gemas laterais brotadas em macieira, cv. Fuji, plantas adultas, na localidade de Luizinho $1080 \mathrm{~m}, 1999$.

\section{REFERÊNCIAS BIBLIOGRÁFICAS}

EREZ, A.; LAVEE, S. The effect of climatic condition development of peach buds: Temperature. Journal of the American Society for Horticultural Science, Alexandria, v.96, n.6, p.711-714, 1971.

EREZ,A.; LAVEE, S. E.; SAMISH, R.M. The effect of limitation in light during the rest period on leaf bud of peach (Prunus persica). Physiologia Plantarum, Copenhagen, v.21, p.759764,1968 .

IUCHI, T.; IUCHI,V.L.. Efeito do arqueamento, anelamento e óleo mineral + DNOC sobre a quebra da dormência da macieira (Malus doméstica Borkh.). Revista Brasileira de Fruticultura., Cruz das Almas, v. 13, n.3, p.139-145, 1991.

IUCHI,T., GUIDONI, A..L.; IUCHI, V.L. Efeito de DNOC e DNBP na presença de óleo mineral sobre a quebra de dormência da macieira (Malus doméstica Borkh.). Revista Brasileira de Fruticultura, Cruz das Almas - BA, v. 12 n. 2, p. 19-26,1990.

IUCHI,T.; OLIVEIRA, J.O.; IUCHI,V.L. Efeito do óleo mineral e DNOC sobre quebra de dormência de macieira (Malus doméstica (Borkh.). Revista Brasileira de Fruticultura., Cruz das Almas,

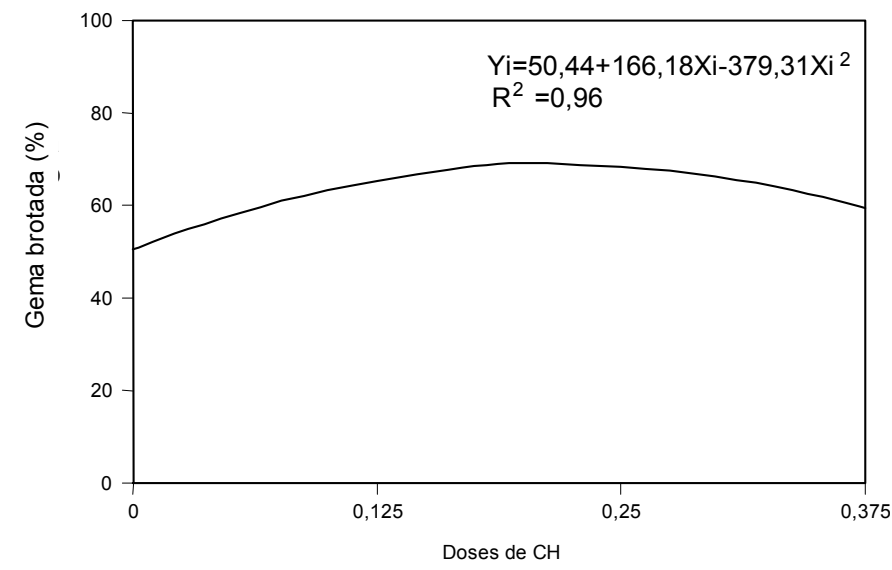

FIGURA 8 - Percentual de gemas brotadas em macieira, cv. Gala, plantas novas, na localidade de Odilon, 1999.

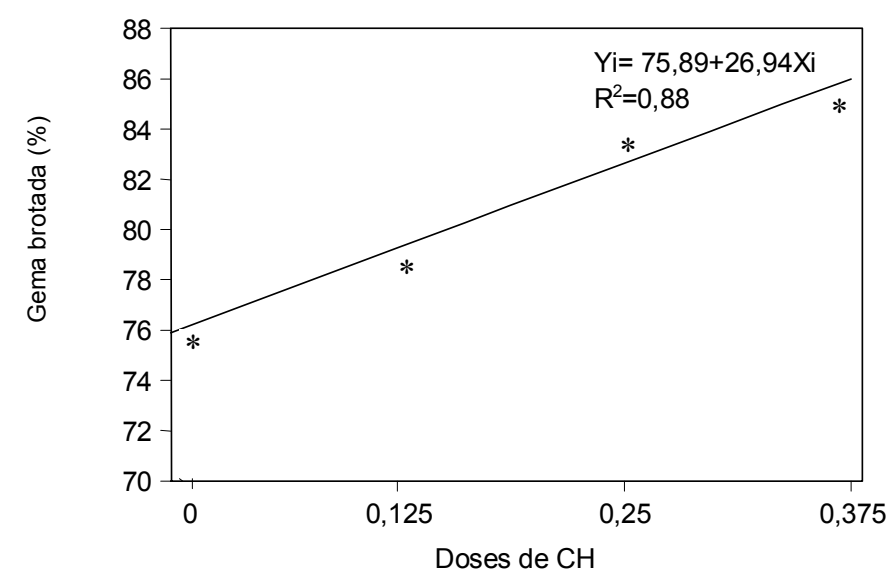

FIGURA 10 - Percentual de gemas laterais brotadas em macieira, cv. Gala, plantas adultas, na localidade de Varzea $930 \mathrm{~m}, 1999$.

v. 9, n. 2, p. $15-21,1987$.

IUCHI, T.; IUCHI, V.L. Efeito da época da mistura de óleo mineral e DNOC sobre a quebra da dormência da macieira (Malus doméstica Borkh.). In: CONGRESSO BRASILEIRO DE FRUTICULTURA, 7., Brasília. Anais..., v. 2, p. 327-335.

IUCHI, T.; IUCHI, V.L.; SAID, J.P. Efeito de óleo mineral e DNOC sobre a quebra da dormência em gemas de ameixeira (Prunus salicina Lindl). Revista Brasileira de Fruticultura, Cruz das Almas, v. 7, p. 59-65, 1985.

NEE, C. C. Overcoming bud dormancy with hidrogen cyanamide: Timing and mechanism. 1986. Diss. (PhD) - Oregon State University, Corvallis, 125 p., 1986.

PASQUAL, M.; PETRI, J. L. Efeito de diferentes sais de dinitro na quebra da dormência da macieira. In: CONGRESSO BRASILEIRO DE FRUTICULTURA, 5., 1979, Pelotas. Anais..., Pelotas. Sociedade Brasileira de Fruticultura, 1979, v. 1, p. 339350 .

PASQUAL, M.; CHALFUN, N.N..; PETRI, J.L; IUCHI, T.; 
FORTES, G.R.L. Efeitos do clima, óleo mineral e dinitro-orthocresol na quebra da dormência da macieira ( Malus communis D.C., Cultivar Golden Delicious. In: CONGRESSO BRASILEIRO DE FRUTICULTURA, 4., 1977,Salvador BA. Anais... Cruz das Almas: Sociedade Brasileira de Fruticultura, 1978.p 213-221.

PETRI, J.; PASQUAL, M.; FORTES, G.R.L.; IUCHI,T.; CAMILO,.A.P.C. Efeito da colaboração de produtos químicos na quebra da dormência da macieira da cultivar Golden Delicious. In: CONGRESSO BRASILEIRO DE FRUTICULTURA. 3., 1975. Rio de Janeiro, R.J. Anais... Campinas: Sociedade Brasileira de Fruticultura, 1976.p. 447-454.
PETRI, J.L. Dormência da macieira. In: EMPASC. Manual da cultura da macieira. Florianópolis,1986.p163-201.

PETRI, J.L.; PALLADINI, L.A.; SHUCK, E.; DUCROQUET, J.H.H.J.; MATOS, C.S. Dormência e indução da brotação de fruteiras de clima temperado. Florianópolis. EPAGRI, 1996.110 p.

SAMISH, R.M.; LAVEE, S.; EREZ, A. The Physiology of rest and its application to fruit growing. Bet. Dagan: The National and University Institute of agriculture, $1967.65 \mathrm{p}$. 